\title{
INTEGRAL REPRESENTATIONS AND REFINEMENT-UNBOUNDEDNESS
}

\author{
WILLIAM D. L. APPLING
}

1. Introduction. Suppose $U$ is a set, $F$ is a field of subsets of $U, \mathfrak{p}$ is the set of all real-valued functions defined on $F, p_{A}$ is the set of all bounded and finitely additive elements of $\mathfrak{p}, \mathfrak{p}^{+}$is the set of all nonnegative-valued elements of $\mathfrak{p}$, and $\mathfrak{p}_{A}^{+}=\mathfrak{p}_{A} \cap \mathfrak{p}^{+}$.

Suppose $\mu$ is in $\mathfrak{p}_{A}^{+}$.

Definition. If $\mathfrak{T}$ is a number set and $\xi$ is in $\mathfrak{p}_{A}$, then the statement that $\xi$ is $\mu$-dense in $\mathfrak{N}$ means that if $V$ is in $F$ and $0<c$, then there is a subdivision $\&$ of $V$ and a function $\mathfrak{n}$ from $\mathbb{E}$ into $\mathfrak{M}$ such that

$$
\sum_{\mathscr{E}}|\xi(I)-\mathfrak{n}(I) \mu(I)|<c .
$$

We prove the following integral representation theorem $(\$ 3)$ :

THEOREM 3.1. If $\mathbb{T r}$ is a bounded number set and $\xi$ is an element of $\mathfrak{p}_{A}$ which is $\mu$-dense in $\mathfrak{T}$, then there is a function $\boldsymbol{\theta}$ from $F$ into $\overline{\mathfrak{T}}$ (i.e., $\mathfrak{M}$ plus its closure) such that if $V$ is in $F$, then the integral (\$2)

$$
\int_{V} \theta(I) \mu(I)
$$

exists and is $\xi(V)$.

The question naturally arises as to necessary and sufficient conditions under which, in the statement of Theorem $3.1, \overline{\mathfrak{M}}$ may be replaced by $\mathscr{T}$. By considering the previously defined [1] notion of refinement-unboundedness (see [1] or $\$ 4$ of this paper), we obtain the following characterization theorem $(\$ 4)$ :

THEOREM 4.1. The following three statements are equivalent:

(1) If $\mathfrak{T}$ is a bounded number set and $\xi$ is an element of $\mathfrak{p}_{A}$ which is $\mu$-dense in $\mathfrak{T}$, then there is a function $\phi$ from $F$ into $\mathfrak{T}$ such that if $V$ is in $F$, then $\int_{V} \phi(I) \mu(I)$ exists and is $\xi(V)$.

(2) If $\mathbb{T}$ is a bounded number set and $\theta$ is a function from $F$ into $\bar{N}$ and $\int_{U} \boldsymbol{\theta}(I) \mu(I)$ exists, then there is a function $\boldsymbol{\phi}$ from $\boldsymbol{F}$ into $\mathbb{T}$ such that if $V$ is in $\boldsymbol{F}$, then $\int_{V} \boldsymbol{\phi}(I) \mu(I)$ exists and is $\int_{V} \boldsymbol{\theta}(I) \mu(I)$.

(3) There is a $\mu$-refinement-unbounded (\$4) element of $\mathfrak{p}^{+}$.

Presented to the Society, April 6, 1967 under the title Refinement unboundedness and integral representations; received by the editor April 19, 1967. 
2. Preliminary theorems and definitions. If $V$ is in $F$, then the statement that $\mathfrak{D}$ is a subdivision of $V$ means that $\mathfrak{D}$ is a finite collection of mutually exclusive sets of $F$ whose union is $V$.

If $\mathfrak{D}$ is a subdivision of a set $V$ of $F$, then the statement that $\mathbb{F}$ is a refinement of $\mathfrak{D}$ means that $\mathbb{E}$ is a subdivision of $V$, every set of which is a subset of some set of $\mathfrak{D}$.

Throughout this paper all integrals considered will be Hellinger [3] type limits (i.e. for refinements of subdivisions) of the appropriate sums.

Suppose $\boldsymbol{\alpha}$ is in $\mathfrak{p}$.

Suppose $(S$ is a subdivision of $U$. If $V$ is in $F$, then the statement that $\boldsymbol{\alpha}$ is $\sum$-bounded on $V$ with respect to $(5)$ means that if $\mathscr{X}=\{z \mid z$ $=\sum \notin \alpha(I)$, $₹$ a subdivision of $V$ and a subset of a refinement of $\left.(\xi)\right\}$, then $-\infty<s_{*}(\alpha)(V)=\inf \mathscr{N} \leqq \sup \Re=s^{*}(\alpha)(V)<\infty$. We adopt the convention that throughout this paper, as in the preceding definition, $s^{*}$ and $s_{*}$ will be understood to be defined in terms of the last mentioned subdivision in the discussion at hand with respect to which the functions under consideration are $\sum$-bounded on $U$. We see that $\boldsymbol{\alpha}$ is $\sum$-bounded on $U$ with respect to $H$ iff for each $V$ in $F, \alpha$ is $\sum$ bounded on $V$ with respect to $\$$, in which case, if $V$ is in $F$ and $F$ is a refinement of each of the subdivisions $\mathfrak{D}$ and $\mathfrak{D}^{\prime}$ of $V$, then

$$
\sum_{\mathscr{D}} s_{*}(\boldsymbol{\alpha})(I) \leqq \sum_{\mathbb{E}} s_{*}(\boldsymbol{\alpha})(I) \leqq \sum_{\mathbb{E}} s^{*}(\boldsymbol{\alpha})(I) \leqq \sum_{\mathscr{D}^{\prime}} s^{*}(\boldsymbol{\alpha})(I),
$$

so that we have the existence and following relationship of the following integrals:

$$
\int_{V} s_{*}(\alpha)(I) \leqq \int_{V} s^{*}(\alpha)(I),
$$

and we see that $\int_{V} \boldsymbol{\alpha}(I)$ exists iff $\int_{V S_{*}}(\boldsymbol{\alpha})(I)=\int_{V} \mathbf{s}^{*}(\boldsymbol{\alpha})(I)$, in which case $\int_{V} S_{*}(\alpha)(I)=\int_{V} \boldsymbol{\alpha}(I)=\int_{V} \mathbf{s}^{*}(\alpha)(I)$.

We observe that $\int_{U} \boldsymbol{\alpha}(I)$ exists iff for each $V$ in $F, \int_{V} \boldsymbol{\alpha}(I)$ exists. We take for granted the linearity and field-wise-additive properties of our in tegrals.

We state without proof a theorem of Kolmogoroff [4]:

Theorem 2.K.1. If $\int_{U} \boldsymbol{\alpha}(I)$ exists, then $\int_{U}\left|\boldsymbol{\alpha}(I)-\int_{I} \boldsymbol{\alpha}(J)\right|=0$.

Suppose each of $\left\{a_{i}\right\}_{i=1}^{n}$ and $\left\{b_{i}\right\}_{i=1}^{n}$ is a number sequence. We have the following two inequalities:

$\min \left\{a_{1}, \cdots, a_{n}\right\}+\min \left\{b_{1}, \cdots, b_{n}\right\} \leqq \min \left\{a_{1}+b_{1}, \cdots, a_{n}+b_{n}\right\}$, 


$$
\left|\min \left\{a_{1}, \cdots, a_{n}\right\}-\min \left\{b_{1}, \cdots, b_{n}\right\}\right| \leqq \sum_{i=1}^{n}\left|a_{i}-b_{i}\right| \cdot
$$

The first of the above inequalities implies that if $\left\{\beta_{i}\right\}_{i=1}^{n}$ is a sequence of elements of $p_{A}^{+}$and $\mathbb{F}$ is a refinement of a subdivision $\mathfrak{D}$ of a set $V$ of $F$, then

$$
0 \leqq \sum_{\mathfrak{E}} \min \left\{\beta_{1}(I), \cdots, \beta_{n}(I)\right\} \leqq \sum_{\mathcal{D}} \min \left\{\beta_{1}(I), \cdots, \beta_{n}(I)\right\},
$$

so that

$$
\int_{V} \min \left\{\beta_{1}(I), \cdots, \beta_{n}(I)\right\}
$$

exists.

An immediate consequence of Theorem 2.K.1 and the second of the above inequalities is the following corollary which we state without proof:

CoROLlaRy 2.K.1. If $\left\{\boldsymbol{\beta}_{i}\right\}_{i=1}^{n}$ is a sequence of elements of $\mathfrak{p}^{+}$such that $\int_{U} \beta_{i}(I)$ exists for $i=1, \cdots, n$, then

$$
\int_{U}\left|\min \left\{\beta_{1}(I), \cdots, \beta_{n}(I)\right\}-\min \left\{\int_{I} \beta_{1}(J), \cdots, \int_{I} \beta_{n}(J)\right\}\right|=0,
$$

so that if $V$ is in $F$, then

$$
\int_{V} \min \left\{\boldsymbol{\beta}_{1}(I), \cdots, \boldsymbol{\beta}_{n}(I)\right\}
$$

exists and is

$$
\int_{V} \min \left\{\int_{I} \beta_{1}(I), \cdots, \int_{I} \beta_{n}(I)\right\} .
$$

If in subsequent statements, the existence of a given integral or its equivalence to a given integral is an immediate consequence of the statements of this section, the integral need only be written and the proof of existence or equivalence left to the reader.

3. The representation theorem. In this section we prove Theorem 3.1 , as stated in the introduction.

Proof of Theorem 3.1. We see that there is a function $\boldsymbol{\beta}$ from $F$ in to $\overline{\mathscr{T}}$ such that if $V$ is in $F$, then

$$
|\xi(V)-\beta(V) \mu(V)|=\inf \{z|z=| \xi(V)-x \mu(V) \mid, x \text { in } \mathfrak{T}\} .
$$


Suppose $0<c$. There is a finite subset $\left\{a_{1}, \cdots, a_{n}\right\}$ of $\mathscr{N}$ such that if $x$ is in $\overline{\mathfrak{T}}$, then $\min \left\{\left|x-a_{1}\right|, \cdots,\left|x-a_{n}\right|\right\}<c /[4(\mu(U)+1)]$.

We see that $\int_{U} \min \left\{\left|\xi(I)-a_{1} \mu(I)\right|, \cdots,\left|\xi(I)-a_{n} \mu(I)\right|\right\}$ exists, since $\int_{U}\left|\xi(I)-a_{i} \mu(I)\right|$ exists for $i=1, \cdots, n$.

There is a function $\gamma$ from $F$ into $\left\{a_{1}, \cdots, a_{n}\right\}$ such that if $I$ is in $F$, then

$$
|\xi(I)-\gamma(I) \mu(I)|=\min \left\{\left|\xi(I)-a_{1} \mu(I)\right|, \cdots,\left|\xi(I)-a_{n} \mu(I)\right|\right\} .
$$

There is a subdivision $\mathfrak{D}$ of $U$ such that if $\mathbb{E}$ is a refinement of $\mathfrak{D}$, then

$$
\left|\int_{U}\right| \xi(I)-\gamma(I) \mu(I)\left|-\sum_{\mathscr{E}}\right| \xi(I)-\gamma(I) \mu(I)||<c / 4 .
$$

For each $I$ in $\mathfrak{D}$ there is a subdivision $\mathbb{E}_{I}$ of $I$ and a function $\boldsymbol{n}_{I}$ from $\xi_{I}$ into $\mathscr{T}$ such that $\sum \mathbb{E}_{I}\left|\xi(J)-n_{I}(J) \mu(J)\right|<c / 4 N$, where $N$ is the number of elements of $\mathfrak{D}$, and there is a function $\lambda_{I}$ from $\mathfrak{E}_{I}$ into $\left\{a_{1}, \cdots, a_{n}\right\}$ such that for each $J$ in $\mathscr{E}_{I}$,

Therefore

$$
\left|\lambda_{I}(J)-n_{I}(J)\right|<c /[4(\mu(U)+1)] \text {. }
$$

$$
\begin{aligned}
& \int_{U}|\xi(J)-\gamma(J) \mu(J)|<c / 4+\sum_{\mathcal{D}} \sum_{\mathbb{E}_{I}}|\xi(J)-\gamma(J) \mu(J)| \\
& \leqq c / 4+\sum_{\mathcal{D}} \sum_{\mathbb{G}_{I}}\left|\xi(J)-\lambda_{I}(J) \mu(J)\right| \\
& \leqq c / 4+\sum_{\mathcal{D}} \sum_{\mathbb{E}_{I}}\left|\xi(J)-\mathbf{n}_{I}(J) \mu(J)\right| \\
&+\sum_{\mathfrak{D}} \sum_{\mathbb{E}_{I}}\left|\mathbf{n}_{I}(J)-\lambda_{I}(J)\right| \mu(J) \\
&< c / 4+N(c / 4 N)+\{c /[4(\mu(U)+1)]\} \mu(U)<3 c / 4 .
\end{aligned}
$$

For each $I$ in $\mathfrak{D}$, there is a subdivision $\xi_{I}^{\prime}$ of $I$ such that $0 \leqq s^{*}(|\xi-\S \mu|)(I)-\sum \mathbb{E}_{I}^{\prime}|\xi(J)-\boldsymbol{\beta}(J) \mu(J)|<c / 16 N$.

Now

$$
\begin{aligned}
& \int_{U} s^{*}(|\xi-\beta \mu|)(I) \leqq \sum_{D} s^{*}(|\xi-\beta \mu|)(I) \\
& \leqq \sum_{\mathscr{D}}\left\{c / 16 N+\sum_{\mathscr{E}_{I}^{\prime}}|\xi(J)-\S(J) \mu(J)|\right\} \\
& \leqq c / 16+\sum_{\mathcal{D}} \sum_{\mathscr{G}_{I}^{\prime}}|\xi(J)-\gamma(J) \mu(J)| \\
&<c / 16+\int_{U}|\xi(J)-\gamma(J) \mu(J)|+c / 4 \\
&<c / 16+3 c / 4+c / 4=17 c / 16 .
\end{aligned}
$$


Therefore $\quad 0 \leqq \int_{U} \boldsymbol{S}_{*}(|\xi-\beta \mu|)(I) \leqq \int_{U} \mathbf{s}^{*}(|\xi-\beta \mu|)(I)=0$, so that $\int_{U}|\xi(I)-\boldsymbol{\beta}(I) \mu(I)|$ exists and is 0 , which we easily see implies that if $V$ is in $F$, then $\int_{V} \boldsymbol{\beta}(I) \mu(I)$ exists and is $\xi(V)$.

4. The characterization theorem. Definition [1]: If $\omega$ is in $p^{+}$, then the statement that $\omega$ is $\mu$-refinement-unbounded means that if $k$ is a positive number, then there is a subdivision $\mathfrak{D}$ of $U$ such that if $I$ is in a refinement of $\mathfrak{D}$ and $\mu(I) \neq 0$, then $\omega(I)>k$.

We state a previous theorem of the author [2].

Theorem 4.A.1. Suppose $\mathrm{o}$ is in $\mathrm{p}^{+}$and that if each of $c$ and $k$ is a positive number, then there is a subdivision $\mathfrak{D}$ of $U$ such that if $\&$ is a refinement of $\mathfrak{D}$, then $\sum \mathfrak{E}^{*} \mu(I)<c$, where $\mathbb{F}^{*}=\{I \mid I$ in $\mathbb{E}, \boldsymbol{\sigma}(I) \leqq k\}$. Then there is a $\mu$-refinement-unbounded element of $\mathrm{p}^{+}$.

We now prove Theorem 4.1, as stated in the introduction.

Proof of Theorem 4.1. We first show that (1) implies (2).

Suppose (1) is true and $\mathscr{T}$ is a bounded number set and $\boldsymbol{\theta}$ is a function from $\boldsymbol{F}$ into $\overline{\mathfrak{M}}$ and $\int_{U} \boldsymbol{\theta}(I) \mu(I)$ exists. Let $\xi$ be the element of $\mathfrak{p}$ defined by $\xi(V)=\int_{V} \boldsymbol{\theta}(I) \mu(I)$. Obviously $\xi$ is in $\mathfrak{p}_{A}$.

We now show that $\xi$ is $\mu$-dense in $\mathbb{T}$.

Suppose $0<c$ and $V$ is in $F$. There is a subdivision $\mathfrak{D}$ of $V$ such that if $\mathcal{E}$ is a refinement of $\mathfrak{D}$, then $\sum_{\mathbb{E}}|\xi(I)-\boldsymbol{\theta}(I) \mu(I)|<c / 2$. For each $I$ in $\mathfrak{D}$, there is a number $\lambda(I)$ in $\mathscr{M}$ such that $|\lambda(I)-\boldsymbol{\theta}(I)|$ $<c /[2(\mu(U)+1)]$. This implies that

$$
\begin{aligned}
\sum_{\mathcal{D}}|\xi(I)-\lambda(I) \mu(I)| & \leqq \sum_{\mathfrak{D}}|\xi(I)-\theta(I) \mu(I)|+\sum_{\mathcal{D}}|\boldsymbol{\theta}(I)-\lambda(I)| \mu(I) \\
& <c / 2+\{c /[2(\mu(U)+1)]\} \mu(U) \leqq c .
\end{aligned}
$$

Therefore $\xi$ is $\mu$-dense in $\mathscr{T}$ and therefore there is a function $\phi$ from $F$ into $\mathbb{T}$ such that if $V$ is in $F$, then $\int_{V} \phi(I) \mu(I)$ exists and is $\int_{V} \boldsymbol{\phi}(I) \mu(I)$.

Therefore (1) implies (2).

It is an immediate consequence of Theorem 3.1 that (2) implies (1). We now show that (2) implies (3).

Suppose (2) is true. Let $\mathscr{T}=\{z \mid z=1 / q, q$ a positive integer $\}$. For each $V$ in $\boldsymbol{F}$, let $\boldsymbol{\theta}(V)=0$. Obviously $\int_{V} \boldsymbol{\theta}(I) \mu(I)=0$ for all $V$ in $\boldsymbol{F}$. Since 0 is in $\overline{\mathscr{M}}$, it follows that there is a function $\phi$ from $F$ into $\mathfrak{M}$ such that if $V$ is in $F$, then $\int_{V} \phi(I) \mu(I)$ exists and is $\int_{V} \theta(I) \mu(I)$.

Now suppose that each of $c$ and $k$ is a positive number. There is a subdivision $\mathfrak{D}$ of $U$ such that if $\mathbb{E}$ is a refinement of $\mathfrak{D}$, then $\sum \mathbb{E} \phi(I) \mu(I)<c / k$, so that $\sum \mathbb{E}^{*} \mu(I)<c$, where $\mathfrak{F}^{*}=\{I \mid I$ in $\mathbb{E}$, $\phi(I) \geqq 1 / k\}=\{I \mid I$ in $\mathcal{E}, 1 / \phi(I) \leqq k\}$. 
It follows that the function $1 / \phi$ satisfies the hypothesis of Theorem 4.A.1, so that there is a $\mu$-refinement-unbounded element of $p^{+}$. Therefore (2) implies (3).

We now show that (3) implies (2). Suppose (3) is true, i.e. that there is a $\mu$-refinement-unbounded element $\omega$ of $\mathfrak{p}^{+}$, and $\mathfrak{T}$ is a bounded number set and $\theta$ is a function from $F$ into $\overline{\mathscr{N}}$ such that $\int_{U} \boldsymbol{\theta}(I) \mu(I)$ exists.

There is a subdivision $\mathfrak{D}^{*}$ of $U$ such that if $I$ is in a refinement of $\mathfrak{D}^{*}$ and $\mu(I) \neq 0$, then $\omega(I)>0$.

There is a function $\phi$ from $F$ into $\mathscr{T}$ such that if $I$ is in a refinement of $\mathfrak{D}^{*}$ and $\mu(I) \neq 0$, then $|\boldsymbol{\theta}(I)-\boldsymbol{\phi}(I)|<1 / \boldsymbol{\omega}(I)$.

Suppose $0<c$ and $V$ is in $F$. There is a subdivision $\mathfrak{D}$ of $V$ such that if $\mathbb{E}$ is a refinement of $\mathfrak{D}$, then $\left|\int_{V} \theta(I) \mu(I)-\sum_{\mathbb{E} \theta}(I) \mu(I)\right|<c / 2$. There is a refinement $\mathfrak{D}^{\prime}$ of $\mathfrak{D}^{*}$ such that if $I$ is in a refinement of $\mathfrak{D}^{\prime}$ and $\mu(I) \neq 0$, then $\omega(I)>2(\mu(U)+1) / c$. There is a subdivision $\mathfrak{D}^{\prime \prime}$ of $V$ which is a refinement of $\mathfrak{D}$ and a subset of a refinement of $\mathfrak{D}^{\prime}$. If $\mathbb{E}$ is a refinement of $\mathfrak{D}^{\prime \prime}$, then

$$
\begin{aligned}
\mid \int_{V} \theta(I) \mu(I) & -\sum_{\mathscr{E}} \phi(I) \mu(I)|\leqq| \int_{V} \theta(I) \mu(I)-\sum_{\mathscr{E}} \theta(I) \mu(I) \mid \\
& +\sum_{\mathbb{E}}|\boldsymbol{\theta}(I)-\phi(I)| \mu(I)<c / 2+\sum_{\mathbb{E}^{*}}[\mu(I) / \omega(I)] \leqq c / 2 \\
& +\{c /[2(\mu(U)+1)]\} \mu(U) \leqq c,
\end{aligned}
$$

where $\mathfrak{F}^{*}=\{I \mid I$ in $\leftleftarrows, \mu(I) \neq 0\}$.

Therefore $\int_{V} \phi(I) \mu(I)$ exists and is $\int_{V} \theta(I) \mu(I)$. Therefore (3) implies (2). Therefore (1), (2) and (3) are equivalent.

\section{REFERENCES}

1. W. D. L. Appling, Refinement-unbounded set functions and absolute continuity, Math. Z. 92 (1966), 140-145.

2. - An extended notion of refinement-unboundedness, Monatsh. Math. 72 (1968), 9-17.

3. E. Hellinger, Die Orthogonalinvarianten quadratischer Formen von unendlichvielen Variablen, Dissertation, Gottingen, 1907.

4. A. Kolmogoroff, Untersuchungen über den Integralbegriff, Math. Ann. 103 (1930), 654-696.

North Texas State University 